\title{
Markus Messling, Marcel Lepper, Jean-Louis Georget, Höhlen. Obsession der Vorgeschichte
}

\section{Guillaume Robin}

\section{OpenEdition}

\section{Journals}

Édition électronique

URL : http://journals.openedition.org/ifha/10346

DOI : $10.4000 /$ ifha. 10346

ISSN : 2198-8943

\section{Éditeur}

IFRA - Institut franco-allemand (sciences historiques et sociales)

Référence électronique

Guillaume Robin, «Markus Messling, Marcel Lepper, Jean-Louis Georget, Höhlen. Obsession der Vorgeschichte», Revue de l'IFHA [En ligne], Date de recension, mis en ligne le 02 octobre 2019, consulté le 24 septembre 2020. URL : http://journals.openedition.org/ifha/10346 ; DOI : https://doi.org/ 10.4000/ifha. 10346

Ce document a été généré automatiquement le 24 septembre 2020.

(C)IFHA 


\title{
Markus Messling, Marcel Lepper, Jean-Louis Georget, Höhlen. Obsession der Vorgeschichte
}

\author{
Guillaume Robin
}

\section{RÉFÉRENCE}

Markus Messling, Marcel Lepper, Jean-Louis Georget, Höhlen. Obsession der Vorgeschichte, Berlin : Matthes \& Seitz, 2019, 222 p., $16 €$

Les grottes fascinent parce qu'elles nous interrogent sur nos origines. Espace protecteur et royaume des ténèbres, abritant les ermites et les créatures étranges, elle peut être aussi source de cauchemar, comme mise à l'écran dans Indiana Jones. Mais la grotte est bien plus que ça. Tel est le fil conducteur de cet ouvrage collectif paru dans la collection Fröhliche Wissenschaft de Matthes \& Seitz, intitulé Grottes - Obsession de la préhistoire et codirigé par Markus Messling, Marcus Lepper et Jean-Louis Georget. C'est une citation d'Oscar Wilde qui ouvre le livre, rappelant que si les hommes des cavernes s'étaient pris moins au sérieux, l'histoire eût été différente. La préhistoire est une obsession, comme le soulignent les co-auteurs de l'ouvrage. En témoigne la polémique raciale déclenchée récemment par la découverte de l'homo naledi dans une grotte au nord-ouest de Johannesburg. L'introduction a le mérite de rappeler que l'histoire des fouilles peut être source de tensions et qu'Humboldt lui-même, quand il voulut emporter des restes humains lors de son expédition dans la grotte d'Ataruipe au Vénézuala, s'était déjà exposé à de virulentes protestations des guides indiens. Les auteurs abordent ici la thématique de la grotte autrement que comme espace protecteur mais comme métaphore. Les grottes ne sont pas seulement des espaces qui se décrivent au plan géographique, spéléologique ou archéologique ; ce sont avant tout des espaces permettant la conscience et la connaissance de soi.

Le long article introductif met en perspective les sept contributions, en rappelant tout d'abord le rôle que les grottes ont joué aux différentes époques. La tendance à explorer 
les grottes s'observe pour la première fois dès le XVII ${ }^{e}$ siècle, bien avant qu'elles ne nourrissent l'imaginaire romantique. On songe ici au film La Lumière Bleue et au rôle magnétique que la grotte, lieu des fantasmes, exerce sur les hommes. C'est elle qui, dépouillée de ses cristaux par les hommes, pousse l'héroïne au suicide. Les grottes ont aussi inspiré des copies, comme celles, désormais fameuses, ramenées au début du XX siècle par Leo Frobenius lors de ses expéditions sahariennes et subsahariennes, thème central du colloque consacré à « Leo Frobenius (1873-1938) : histoire croisée de la constitution et de l'appropriation d'un savoir ethnologique en France et en Allemagne » organisé en 2014 par Hélène Ivanoff, Richard Kuba et Jean-Louis Georget.

L'introduction de l'ouvrage met en avant les grottes comme sources d'inspiration pour les artistes au XXe siècle, en particulier les surréalistes en quête de nouvelles formes d'expression. L'art dit « primitif » joua un rôle déterminant pour les avant-gardes et l'art moderne qui s'en inspira largement. Les co-auteurs posent enfin la question plus vaste des conditions de préservation du patrimoine et d'ouverture au public, comme l'illustrent la création d'une réplique de la grotte Chauvet en 2015 ou encore la mise en place expérimentale d'un tirage au sort pour visiter celles d'Altamira pour en faciliter la conservation. La grotte, plus généralement, nous interroge sur la problématique de la conservation des biens culturels et invite à réfléchir sur le processus de construction muséale, à l'instar du projet artistique d'Adalbert Hoesle en 2004, célébrant le $50^{\mathrm{e}}$ anniversaire de la Convention de La Haye pour la protection des biens culturels en cas de conflit armé. Ainsi, c'est dans une archive souterraine abritée par une ancienne mine que 50 conteneurs d'œuvres d'art contemporain furent entreposés pour 1500 ans. Ils ne seront rouverts au public qu'en 3504 .

L'article de Horst Bredekamp rappelle l'exposition « Kunst der Vorzeit » qui s'est tenue en 2016 au Martin Gropius Bau et où furent exposées les immenses toiles-copies de Frobenius. H. Bredekamp souligne l'influence de la peinture pariétale sur l'art moderne mais aussi sur l'expressionnisme abstrait. Ceux-ci, loin de rompre avec tout modèle, réussirent au contraire à jeter des ponts au plan stylistique avec l'art pariétal. Particulièrement dense est la contribution de Franck Hofmann qui commence par rappeler que le romantisme du XIX ${ }^{e}$ siècle considéra la grotte comme un contre-monde poétique à l'intérieur de la société bourgeoise. La grotte-cloaque est aussi un lieu de l'informe dans lequel il faut aussi voir, comme chez Leiris, des parallèles avec les cavités humaines telles que la bouche et l'anus. Le corps peut être vu comme une grotte, une béance dont s'échappent le cri et la souffrance, loin de l'image idéale d'un corps debout. Mais c'est aussi une source de réflexion sur les nouvelles approches curatoriales et les mécanismes de perception, à l'instar de l'installation trompe-l'œil réalisée pour la fondation Prada par Thomas Demand intitulée Processo grottesco. Cette œuvre donnant l'illusion d'une grotte où sont reproduites ses stalactites et ses stalagmites nous interroge par extension sur le processus de création artistique. La grotte atelier transforme l'espace muséal davantage en lieu de production artistique qu'en lieu d'exposition.

Maria Stavrinaki se focalise quant à elle sur l'importance symbolique que la grotte joua pour Georges Bataille. Dans La Naissance de l'art (1955), Bataille interpréta les peintures de Lascaux comme un tournant marquant le point de départ de l'humanité, l'homme cessant à partir de ce « miracle » historique d'être un animal. Ce processus d'humanisation commence avec la mise en scène narrative des peintures. Cependant, ce n'est pas tant en peignant l'animal que l'homme cesse d'être animal lui-même qu'en attribuant à l'animal une forme poétique. Stavrinaki dresse un parallèle entre Bataille 
et Frobenius qui considéra, lui-aussi, la préhistoire comme un âge d'or. Elle nous explique que Bataille, à la différence de la plupart des spécialistes de la préhistoire, n'analyse pas les peintures pariétales de Lascaux en termes de reproduction mais comme un "rituel matriciel », un rituel originel quasi magique marquant le passage de l'animal au stade d'homme. Les peintures de Lascaux, à ce titre, doivent être envisagées chez Bataille comme le début de l'histoire universelle, Hiroshima la fin. Retenons aussi l'article de Cord Riechelmann - réflexion sur l'œuvre de Hans Blumenberg - et celui de Gunter Gebauer chez qui l'importance de l'art pariétal ne réside pas tant dans sa maîtrise formelle que dans sa capacité à extérioriser la pensée réflexive. L'art pariétal représente selon lui un nouveau stade de l'humanité. Les peintures de chasse, comme le souligne G. Gebauer, n'ont pas vocation à représenter la réalité - corroborant ainsi les propos de l'anthropologue André Leroi-Gourhan qui était parvenu à cette conclusion en observant les peintures de Lascaux - mais à manifester sous une forme symbolique les relations entre les hommes. Les peintures des grottes font avant tout prendre conscience à l'homme de son intégration sociale dans un espace concret. Les représentations symboliques de la grotte sont « une métaphore du monde organisé » des hommes.

L'ouvrage est complété par un article de M. Maldovinos sur l'intégration des objets ethnographiques des cultures indigènes mexicaines dans les institutions muséales et le rôle des usages linguistiques dans le processus de classification muséale. Il se conclut par un article de J. Trabant qui appréhende les grottes comme des lieux de l'origine du langage et décrypte les termes utilisées par le philosophe italien Giambattista Vico qui projeta dans les grottes sa vision fantasque et vit en elles un espace de domestication des pulsions animales.

Pour conclure, cet ouvrage collectif jette une nouvelle lumière sur le monde obscur des grottes et les fait renaitre. Ce n'est sans doute pas un hasard si dans le roman de Michel Tournier Robinson ou les Limbes du Pacifique, la grotte représente une renaissance pour Robinson. Il s'introduit dedans en position fœtale comme dans un utérus, le corps enduit de lait, pour faire corps avec la terre. C'est aussi l'explosion de la grotte - mort symbolique de l'ancien Robinson, le civilisateur - qui détruira tout ce que Robinson avait bâti et le rattachait à son humanité. Cet ouvrage collectif apporte de nouveaux éclairages sur la grotte en tant que surface de projection. Faisant écho au numéro d' Allemagne d'Aujourd'hui consacré à l'Allemagne et l'Afrique (2016), il s'inscrit autant qu'il les complète dans le prolongement des travaux sur l'art rupestre africain et des récents ouvrages comme Saisir le terrain ou l'invention des sciences empiriques en France et en Allemagne (2017) et Cercles culturels - Leo Frobenius et son temps (2016). 